Provided for non-commercial research and education use. Not for reproduction, distribution or commercial use.

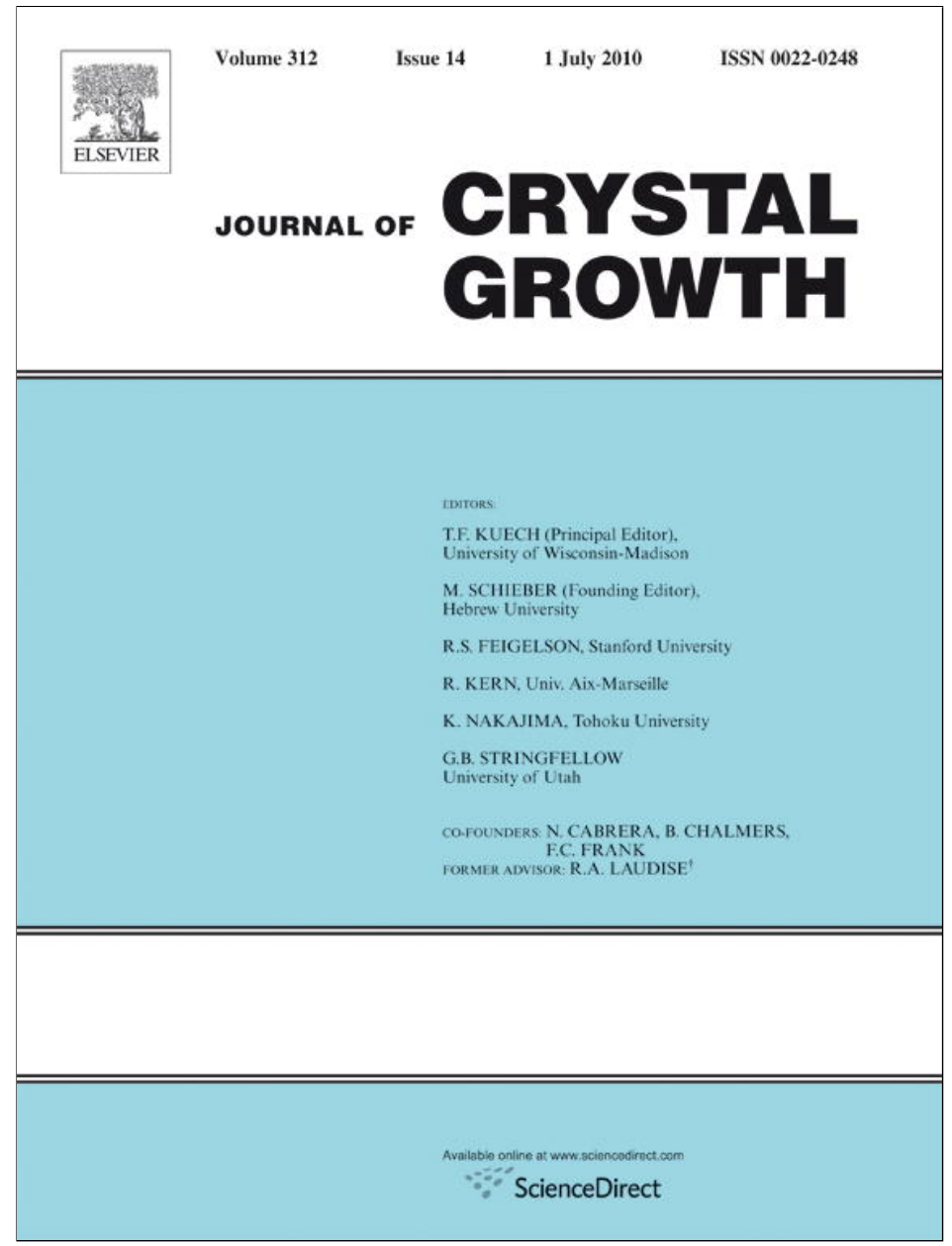

This article appeared in a journal published by Elsevier. The attached copy is furnished to the author for internal non-commercial research and education use, including for instruction at the authors institution and sharing with colleagues.

Other uses, including reproduction and distribution, or selling or licensing copies, or posting to personal, institutional or third party websites are prohibited.

In most cases authors are permitted to post their version of the article (e.g. in Word or Tex form) to their personal website or institutional repository. Authors requiring further information regarding Elsevier's archiving and manuscript policies are encouraged to visit:

http://www.elsevier.com/copyright 


\title{
Selection criterion for the growing dendritic tip in a non-isothermal binary system under forced convective flow
}

\author{
D.V. Alexandrov ${ }^{a}$, P.K. Galenko ${ }^{\text {b,c,*}}$, D.M. Herlach ${ }^{\text {b }}$ \\ a Department of Mathematical Physics, Urals State University, Ekaterinburg 620083, Russian Federation \\ ${ }^{\mathrm{b}}$ Institut für Materialphysik im Weltraum, Deutsches Zentrum für Luft- und Raumfahrt (DLR), 51170 Köln, Germany \\ ${ }^{\mathrm{c}}$ Institut für Festkörperphysik, Ruhr-Universität Bochum, 44780 Bochum, Germany
}

\section{A R T I C L E I N F O}

\section{Article history:}

Received 8 October 2009

Accepted 29 March 2010

Communicated by J.J. Derby

Available online 3 April 2010

Keywords:

A1. Stability

A1. Convection

A1. Dendrite

A1. Solidification

A1. Model

\begin{abstract}
A B S T R A C T
A free dendrite growth during solidification into external forced flow is analyzed using a sharp interface model. A criterion for selection of the stable growth mode is derived for the axisymmetric dendrite growing into non-isothermal binary system under convective flow. The criterion obtained rallies analytic results for dendrite growth under forced convection in a pure system [Ph. Bouissou, P. Pelce, Phys. Rev. A 40 (1989) 6673] and dendrite growth in a stagnant binary system [M. Ben Amar, P. Pelce, Phys. Rev. A 39 (1989) 4263].
\end{abstract}

(c) 2010 Elsevier B.V. All rights reserved.

\section{Introduction}

Dendrite growth controls the microstructure evolution during solidification of melts and solutions [1-3]. Together with quantitative experimental observation of the dynamics and morphology of dendrite growth [4-6,8], new results on theoretical modeling based on sharp- and diffuse-interface methods were obtained to verify the ideas underlying the main concepts of dendrite growth $[4,6,8-10]$. Among these recent problems, the tip kinetics during free dendritic growth into undercooled liquid [7-9] and effect of liquid convection on the mechanism and mode selection of dendritic growth [10] were intensively analyzed from both experimental and theoretical points.

One of the theoretically and practically important problems is in obtaining stable mode of the growing dendrite. The problem of finding a stability criterion for the growing dendrite has deep scientific roots coming from the stability tests of the first analytical solution given by Ivantsov [11-13]. After establishing robust stable conditions for the dendritic tip growing into a onecomponent stagnant liquid $[14,15]$, these were extended to the one-component dendritic growth under forced flow [16-18] as well as to the binary dendritic growth in stagnant media [19]. However, in many cases, knowledge of stable dendritic growth in binary system mode is a crucial question in evaluation and

\footnotetext{
* Corresponding author at: Institut für Materialphysik im Weltraum, Deutsches Zentrum für Luft- und Raumfahrt (DLR), 51170 Köln, Germany. Fax: +4922036012255

E-mail address: Peter.Galenko@dlr.de (P.K. Galenko).
}

verification of the dendritic theory predictions in comparison with experimental data [20]. Therefore, the present analytical investigation is devoted to the problem of a selection criterion for the crystalline dendritic tip growing into a binary liquid under forced convective flow.

The Article is organized as follows. A statement of the problem together with analytical solution in the Oseen approximation is given in Section 2 for the growing binary dendrite under forced convective motion. Section 3 deals with the stability analysis in linear approximation to obtain the dispersion law of the growing dendrite. The latter one defines the critical wave-number selecting marginal mode between degenerating and growing perturbations at the dendritic surface around its tip in the presence of anisotropy of surface energy. The main result of the present analysis is found in Section 4 as a selection criterion given by the solvability condition for the problem. A short discussion about the obtained stable mode for the growing dendritic tip is presented in Section 5 in comparison with previously found criteria for one-component and binary growing dendrites. Finally, Section 6 gives a summary of our conclusions.

\section{The model}

\subsection{Governing equations}

Let us consider a forced convection heat and momentum transfer problem when the crystal interface is assumed to be rough so that the Gibbs-Thomson relation determines the 
interface temperature as a function of the local curvature $1 / R$ of the solid-liquid interface

$T_{i}=T_{0}-\frac{T_{0} \sigma}{Q R}$,

where $T_{0}$ is the crystallization temperature of the pure liquid, $\sigma$ the effective anisotropic liquid-solid surface tension, and $Q$ the latent heat per unit volume of solid.

The temperature field satisfies the diffusion equation in the solid and liquid phases

$\frac{\partial T_{s}}{\partial t}=D_{T} \Delta T_{s}, \quad \frac{\partial T_{l}}{\partial t}+(\vec{w} \cdot \nabla) T_{l}=D_{T} \Delta T_{l}$,

and the concentration of impurities satisfies

$\frac{\partial C_{l}}{\partial t}+(\vec{w} \cdot \nabla) C_{l}=D_{C} \Delta C_{l}$

in the liquid only. Here the advection of heat and mass by the fluid velocity field $\vec{w}$ is taken into account, $D_{T}$ and $D_{C}$ are the diffusive coefficients for temperature and concentration, respectively, $t$ the time, and subscripts $s$ and $l$ designate solid and liquid phases, respectively.

At the interface, the temperature continuity holds. Also, the conservation of heat and impurities must be satisfied, i.e.

$T_{l}=T_{i}-m C_{l}, \quad T_{s}=T_{l}, \quad Q \vec{v} \cdot \vec{n}=D_{T} c_{p}\left(\nabla T_{s}-\nabla T_{l}\right) \cdot \vec{n}$,

$C_{l}\left(1-k_{0}\right) \vec{v} \cdot \vec{n}+D_{C}(\vec{n} \cdot \nabla) C_{l}=0$,

where $\vec{v}$ is the solidification velocity directed along the normal vector to the interface, $c_{p}$ the heat capacity, $k_{0}$ the equilibrium partition coefficient and $m$ the liquidus slope.

The velocity field $\vec{w}$ in the case of the small Reynolds number limit under consideration is described by the so-called Oseen and mass conservation equations [21]

$U \frac{\partial \vec{w}}{\partial z}=-\frac{1}{\rho_{1}} \nabla p+v \Delta \vec{w}, \quad \nabla \cdot \vec{w}=0$,

where $U$ is the external flow velocity far ahead of the crystal, $\rho_{1}$ and $v$ density and kinematic viscosity of the liquid, respectively.

\subsection{Steady-state solution}

The two-dimensional solid-liquid interface of the growing crystal is assumed parabolic, of tip radius $\rho$, and moves at the velocity $V$ along the $z$-direction. The external flow at infinity is parallel to the $\mathrm{Oz}$ axis and is directed to the crystal. Consequently, we consider a case of the so-called "up-stream branch forced flow". The Cartesian coordinates $z$ and $x$ of the growth and transverse directions, respectively, can be written in terms of the parabolic coordinates $\xi$ and $\eta$ as follows:

$z=\frac{\rho}{2}(\eta-\xi), \quad x=\frac{\rho}{2} \sqrt{\xi \eta}$,

where the crystal surface $\eta=1$ has a tip radius $\rho$.

Solution for the velocity field can be easily found in the Oseen approximation. Omitting tedious and cumbersome mathematical manipulations (for details, see Ref. [22]), one can find the following expressions for the components $u_{\xi}$ and $u_{\eta}$ of the velocity field:

$u_{\xi}=\frac{\sqrt{\xi \eta}}{\sqrt{\xi+\eta}} \frac{d f}{d \eta}, \quad u_{\eta}=-\frac{f(\eta)}{2 \sqrt{\xi+\eta}}$,

where

$f(\eta)=2(U+V) \sqrt{\eta}-2 U g(\eta)$, and

$g(\eta)=\sqrt{\eta} \frac{\operatorname{erfc}(\sqrt{\mathfrak{R} \eta / 2})}{\operatorname{erfc}(\sqrt{\mathfrak{R} / 2})}+\frac{\sqrt{2 /(\pi \Re)}}{\operatorname{erfc}(\sqrt{\mathfrak{R} / 2})}[\exp (-\mathfrak{R} / 2)-\exp (-\mathfrak{R} \eta / 2)]$,

with $\mathfrak{R}=\rho U / v$ the Reynolds number.

Using Eqs. (7) and (8), one can integrate the Eqs. (2) and (3) of heat and mass transport in the liquid in their steady-state approximation. Seeking for a simple solution depending on $\eta$ only and rewriting Eqs. (2) and (3) supplemented by the boundary conditions (4) and (5) in parabolic coordinates (see Ref. [16]), we arrive at

$u_{\eta} \frac{d T_{l}}{d \eta}=\frac{2 D_{T}}{\rho \sqrt{\xi+\eta}}\left(\sqrt{\eta} \frac{d^{2} T_{l}}{d \eta^{2}}+\frac{1}{2 \sqrt{\eta}} \frac{d T_{l}}{d \eta}\right), \quad\left(\frac{d T_{l}}{d \eta}\right)_{\eta=1}=-\frac{Q}{c_{p}} \frac{\rho V}{2 D_{T}}$,

$u_{\eta} \frac{d C_{l}}{d \eta}=\frac{2 D_{C}}{\rho \sqrt{\xi+\eta}}\left(\sqrt{\eta} \frac{d^{2} C_{l}}{d \eta^{2}}+\frac{1}{2 \sqrt{\eta}} \frac{d C_{l}}{d \eta}\right), \quad\left(\frac{d C_{l}}{d \eta}\right)_{\eta=1}=-\left(1-k_{0}\right) C_{i} \frac{\rho V}{2 D_{C}}$,

where $C_{i}$ is the concentration at the solid-liquid interface. From Eqs. (11) and (12), solutions for temperature and concentration fields are

$T_{l}(\eta)=T_{i}+\left(T_{\infty}-T_{i}\right) \frac{I(\eta)}{I(\infty)}, \quad C_{l}(\eta)=C_{i}+\left(C_{\infty}-C_{i}\right) \frac{I_{1}(\eta)}{I_{1}(\infty)}$,

with

$I(\eta)=\int_{1}^{\eta} \exp \left[P_{f} \int_{1}^{\eta^{\prime}} \frac{g\left(\eta^{\prime \prime}\right)}{\sqrt{\eta^{\prime \prime}}} d \eta^{\prime \prime}-\left(P_{f}+P_{g}\right) \eta^{\prime}\right] \frac{d \eta^{\prime}}{\sqrt{\eta^{\prime}}}$,

$I_{1}(\eta)=\int_{1}^{\eta} \exp \left[P_{f} \frac{D_{T}}{D_{C}} \int_{1}^{\eta^{\prime}} \frac{g\left(\eta^{\prime \prime}\right)}{\sqrt{\eta^{\prime \prime}}} d \eta^{\prime \prime}-\left(P_{f}+P_{g}\right) \frac{D_{T}}{D_{C}} \eta^{\prime}\right] \frac{d \eta^{\prime}}{\sqrt{\eta^{\prime}}}$,

$P_{g}=\frac{\rho V}{2 D_{T}}, \quad P_{f}=\frac{\rho U}{2 D_{T}}$,

$T_{i}=T_{\infty}+\frac{Q}{c_{p}} P_{g} \exp \left(P_{f}+P_{g}\right) I(\infty)$,

$C_{i}=\frac{C_{\infty}}{1-\left(1-k_{0}\right) \exp \left[\left(P_{f}+P_{g}\right) D_{T} / D_{C}\right] P_{g} I_{1}(\infty) D_{T} / D_{C}}$,

where $P_{g}$ and $P_{f}$ are the Peclet numbers related to the processes of growth and flow, respectively, $T_{\infty}$ and $C_{\infty}$ are the temperature and concentration in the liquid fixed far from the solid-liquid interface, respectively.

It is noteworthy that the Ivantsov parabolas are no longer solutions of the free-boundary problem when surface tension effects are taken into account. However, in the case of weak surface tension effects, steady solutions can be found close to an Ivantsov parabola if a solvability condition is satisfied $[18,23]$. We use here the solvability condition previously derived by Pelce and Bensimon [23] as the vanishing of an oscillating integral in the form

$\int_{-\infty}^{+\infty} G\left[X_{0}(l)\right] Y_{m}(l) d l=0, \quad Y_{m}(l)=\exp \left[i \int_{0}^{l} k_{m}\left(l_{1}\right) d l_{1}\right]$.

The main interest of this formulation is that it is adaptable to other kinds of fronts, e.g., to the Saffman-Taylor finger [17]. One needs the curvature operator $G$ and a continuum of solutions $X_{0}(l)$ from which the function $k_{m}(l)$ of the local non-zero marginal mode of the conjugate dispersion equation for the perturbations is deduced. The condition of application of the solvability criterion is that $k_{m}(l)$ is large compared to the inverse of the scale of the unperturbed solution. It is obtained using Wentzel-KramersBrillouin (WKB) approximation which has been applied to the 
flame propagation [24] and dendritic growth [25]. In other related problems, e.g., in description of the Saffman-Taylor fingers $[26,27]$, it has been shown that one can get non-trivial solvability condition by considering elements $Y_{m}(l)$ (they vary on a wavelength scale $\lambda$, which is small compared to the tip radius of the parabola). In the next section we find these elements in the presence of a forced flow within the framework of the Oseen equations.

\section{Linear stability analysis}

We use the linear stability analysis provided by Bouissou and Pelce [18] in which the growth rate of a perturbation has a wavelength small compared to the characteristic spatial scale of the unperturbed solution. We consider that a perturbation disturbs the fluid on a distance of the order of $\lambda$. The latter enables us to expand the stationary velocity components (8) in a series in $\eta-1$ around the parabola $\eta=1$. Taking into account only the main contributions, we arrive at

$u_{\xi}=\sqrt{\frac{\xi}{1+\xi}}[V+a(\Re) U(\eta-1)], \quad u_{\eta}=-\frac{V}{\sqrt{1+\xi}}$,

where

$a(\mathfrak{R})=\sqrt{\frac{\mathfrak{R}}{2 \pi}} \frac{\exp (-\mathfrak{R} / 2)}{\operatorname{erfc}(\sqrt{\mathfrak{R} / 2})}$.

Hence, from Eqs. (16) and (17) it follows that only the tangent fluid velocity $u_{\xi}$ is dependent of the forced flow close to the tip of the growing parabola.

For the following analysis, we use new local Cartesian coordinates $\left(x_{c}, y_{c}\right)$ fixed to the crystal, where $x_{c}$ and $y_{c}$ are, respectively, the tangent and normal axes to the solid-liquid interface at a point where the normal to the interface has an angle $\theta$ with the growth axis. These coordinates enables us to rewrite the velocity components (16) in the form of a shear flow whose magnitude is a function of $\theta$ as

$\bar{u}=-V \sin \theta-\frac{a U}{\rho} \sin \theta \cos \theta y_{c}, \quad \bar{v}=-V \cos \theta$,

where $\bar{u}$ and $\bar{v}$ designate the tangent and normal velocity components to the interface. Expressing temperature and concentration derivatives from (4) and (5) as

$\frac{d T_{l}}{d y_{c}}=\frac{Q \bar{v}}{D_{T} c_{p}}, \quad \frac{d C_{l}}{d y_{c}}=\frac{C_{i}\left(1-k_{0}\right) \bar{v}}{D_{C}} \quad$ at $y_{c}=0$.

A similar expansion in series for the temperature and concentration fields in the liquid is obtained as follows:

$\overline{T_{l}}=T_{0}-\frac{Q V}{D_{T} c_{p}} \cos \theta y_{c}, \quad \overline{C_{l}}=C_{i}-\frac{C_{i}\left(1-k_{0}\right) V}{D_{C}} \cos \theta y_{c}$.

Let us now pay our attention to the linear stability theory of the aforementioned problem. Let $u^{\prime}, v^{\prime}$ and $T^{\prime}$ designate the perturbations of the stationary field. $\xi^{\prime}$ corresponds to the perturbation of the steady solid-liquid interface with a wavelength $\lambda$ assumed very small compared to $\rho$. The solutions of the perturbed temperature conductivity Eq. (2) in the solid and hydrodynamic Eq. (6) within the framework of the Oseen approximation can be written in the form

$u^{\prime}=-i \varepsilon \omega \sum \exp \left(\omega t+i k x_{c}-\varepsilon k y_{c}\right), \quad v^{\prime}=-\omega \Sigma \exp \left(\omega t+i k x_{c}-\varepsilon k y_{c}\right)$,

$\xi^{\prime}=\Sigma \exp \left(\omega t+i k x_{c}-\varepsilon k y_{c}\right), \quad T_{s}^{\prime}=T_{s 0} \exp \left(\omega t+i k x_{c}-\varepsilon k y_{c}\right)$,

where a relation $v^{\prime}=-\partial \xi^{\prime} / \partial t$ at the solid-liquid interface between perturbations is taken into consideration. Here $\omega$ and $k$ represent the frequency and wave-number of the perturbations, respectively, parameter $\varepsilon$ has the same sign as real part of $k$ since all perturbations cannot diverge as $y_{c}$ goes to $+\infty, \Sigma$ and $T_{s}$ are the perturbation amplitudes of the interface and temperature field, respectively, in the solid.

Consider the perturbed form of nonlinear equation for the temperature in the liquid. Keeping in mind only linear terms in perturbations, one obtains

$\frac{\partial T_{l}^{\prime}}{\partial t}+\bar{u} \frac{\partial T_{l}^{\prime}}{\partial x_{c}}+\bar{v} \frac{\partial T_{l}^{\prime}}{\partial y_{c}}+v^{\prime} \frac{d T_{l}}{d y_{c}}=D_{T}\left(\frac{\partial^{2} T_{l}^{\prime}}{\partial x_{c}^{2}}+\frac{\partial^{2} T_{l}^{\prime}}{\partial y_{c}^{2}}\right)$.

If the forced flow is negligible, the solution has the similar form to $T_{s}^{\prime}$ at large $k$ consistent with the well-known Mullins-Sekerka criterion [12] for $k$ within the framework of the thermal problem of solidification of a pure melt (see, among others, [18]). Substituting

$T_{l}^{\prime}=g\left(y_{c}\right) \exp \left(\omega t+i k x_{c}-\varepsilon k y_{c}\right)$

into Eq. (22) and taking into account Eq. (20), one can get the following equation for the new amplitude function $g\left(y_{c}\right)$ :

$\frac{d^{2} g}{d y_{c}^{2}}-2 \varepsilon k \frac{d g}{d y_{c}}=L\left(g\left(y_{c}\right), y_{c}\right)$,

where

$L\left(g\left(y_{c}\right), y_{c}\right)=\left[\omega+k V \varepsilon \exp (-i \varepsilon \theta)-\frac{a U k \sin \theta \cos \theta}{\rho} y_{c}\right] \frac{g\left(y_{c}\right)}{D_{T}}+\frac{\omega Q V \cos \theta}{c_{p} D_{T}^{2}} \Sigma$.

We search for a solution of Eq. (24) around the MullinsSekerka solution [12] with a constant amplitude $g\left(y_{c}\right)=T_{l 0}=$ const. Substitution $T_{l 0}$ in the right-hand side of (24) gives the first order approximation for $g\left(y_{c}\right)$. The result is

$$
\begin{aligned}
g\left(y_{c}\right)= & T_{l 0}-\left[\left(\frac{\omega}{2 \varepsilon k}+\frac{V}{2} \exp (-i \varepsilon \theta)-\frac{a U \sin \theta \cos \theta}{4 k \rho} i\right) \frac{T_{l 0}}{D_{T}}\right. \\
& \left.+\frac{\omega Q V \cos \theta}{2 D_{T}^{2} c_{p} \varepsilon k} \Sigma\right] y_{c}+\frac{a U \sin \theta \cos \theta}{4 \varepsilon \rho D_{T}} i T_{l 0} y_{c}^{2},
\end{aligned}
$$

where the strong inequality $V / D_{T} \ll k$ is taken into account (we estimate $k$ from the Mullins-Sekerka theory as $10^{7} \mathrm{~m}^{-1}$ [12] and $V / D_{T}$ as $10^{2} \mathrm{~m}^{-1}$ for metallic binary alloys).

Eq. (3) written for the concentration perturbations $C_{l}^{\prime}$ in the liquid can be solved in the same manner. The result is

$C_{l}^{\prime}=h\left(y_{c}\right) \exp \left(\omega t+i k x_{c}-\varepsilon k y_{c}\right)$,

$$
\begin{aligned}
h\left(y_{c}\right)= & C_{l 0}-\frac{a U k \sin \theta \cos \theta}{2 \rho D_{C}\left(V \cos \theta / D_{C}-2 \varepsilon k\right)} i C_{l 0} y_{c}^{2} \\
& +\left\{\left[\omega+V k \varepsilon \exp (-i \varepsilon \theta)+\frac{a U k \sin \theta \cos \theta}{V \cos \theta / D_{C}-2 \varepsilon k} \frac{i}{\rho}\right] \frac{C_{l 0}}{D_{C}}\right. \\
& \left.+\frac{\omega C_{i}\left(1-k_{0}\right) V \cos \theta}{D_{C}^{2}} \Sigma\right\} \frac{y_{c}}{V \cos \theta / D_{C}-2 \varepsilon k} .
\end{aligned}
$$

Now, expanding the boundary conditions (4) and (5) in a series, we arrive at the following set of conditions imposed at the solidliquid interface $y_{c}=0$ :

$T_{l}^{\prime}=\frac{Q V \cos \theta}{D_{T} c_{p}} \xi^{\prime}-m C_{l}^{\prime}+\frac{m C_{i}\left(1-k_{0}\right) V \cos \theta}{D_{C}} \xi^{\prime}-\frac{Q d}{c_{p}} \frac{\partial^{2} \xi^{\prime}}{\partial y_{c}^{2}}$,

$T_{s}^{\prime}=m C_{l}^{\prime}-\frac{m C_{i}\left(1-k_{0}\right) V \cos \theta}{D_{C}} \xi^{\prime}+\frac{Q d}{c_{p}} \frac{\partial^{2} \xi^{\prime}}{\partial y_{c}^{2}}$,

$\frac{Q}{c_{p}} \frac{\partial \xi^{\prime}}{\partial t}=D_{T}\left(\frac{\partial T_{s}^{\prime}}{\partial y_{c}}-\frac{\partial T_{l}^{\prime}}{\partial y_{c}}-\frac{Q V^{2} \cos ^{2} \theta}{D_{T}^{2} c_{p}} \xi^{\prime}\right)$, 
$\frac{1-k_{0}}{D_{C}}\left(C_{i} v^{\prime}-V \cos \theta C_{l}^{\prime}\right)=\frac{\partial C_{l}^{\prime}}{\partial y_{c}}+\frac{C_{i} k_{0}\left(1-k_{0}\right) V^{2} \cos ^{2} \theta}{D_{C}^{2}} \xi^{\prime}$,

where $d=\sigma c_{p} T_{0} / Q^{2}$ stands for the capillary length. Substitution of perturbations (21), (23) and (27) into the boundary conditions (28) gives four linear relations for the perturbation amplitudes $\Sigma$, $T_{l 0}, T_{s}$, and $C_{l 0}$.

To emphasize the method of deduction of the dispersion relation from Eq. (28), consider a frame with the normal axis and tangent axis to the interface and whose origin moves normally to the solid-liquid interface at the velocity $V \cos \theta$. Because of the rotational symmetry of the system, a perturbation of wave number $k$ grows with the rate $\omega(k)$. If now the origin of the frame moves in the $z$-direction with the constant velocity $V$, the growth rate of the same perturbation is $\omega(k)+i V k \sin \theta$ due to the tangential velocity of the new frame $V \sin \theta$ [19]. Therefore, replacing $\omega(k)$ by $-i V k \sin \theta$ at the neutral stability curve and eliminating the perturbation amplitudes from expressions (28), we arrive at the following equation for the wave number $k$ :

$$
\begin{aligned}
k^{2}= & \left(\frac{V}{2 d D_{T}}+\frac{m C_{i}\left(1-k_{0}\right) V}{d\left(Q / c_{p}\right) D_{C}}\right) \exp (i \theta)-\frac{a U \sin \theta \cos \theta}{8 \rho D_{T}} i \\
& +\frac{m C_{i}\left(1-k_{0}\right) a U V \sin \theta \cos \theta}{8 d\left(Q / c_{p}\right) \rho D_{C}^{2} k^{2}}\left(\frac{2 V \cos ^{2} \theta}{D_{C} k} i-\sin \theta\right),
\end{aligned}
$$

where, in accordance with our estimates $k \sim 10^{7} \mathrm{~m}^{-1}$, $V / D_{T} \sim 10^{2} \mathrm{~m}^{-1}, V / D_{C} \sim 10^{6} \mathrm{~m}^{-1}, U \sim V, d \sim 10^{-10} \mathrm{~m}, \rho \sim 10^{-5} \mathrm{~m}$, we write down only the terms corresponding to the solution in the absence of a forced flow and terms describing the influence of this flow for pure thermal and impurity problems. The first term in the right-hand side of Eq. (29) is the solution of the thermal problem without impurities and external flow [17,18,23], the first two summands give the solution of the thermal problem complicated by impurity transport in the liquid without external flow [19], the first and third summands describe the solution with a forced flow in the absence of the solute transport [18]. As a result, solution of the complete Eq. (29) gives the critical wave number with which perturbations neither grow or decay at the dendrite tip growing into the binary system under convective flow.

\section{Solvability condition and stability criterion}

Now, we consider the case of a fourfold symmetry of the crystal. Therefore, the capillary length can be written as $d(\theta)=d_{0}(1-\beta \cos 4 \theta)$, where $\beta=15 \varepsilon_{c} \ll 1$ is the anisotropic factor, $\varepsilon_{c}$ is the strength of anisotropy of the surface energy at the solidliquid interface, and $d_{0}$ the capillary constant.

Substituting the wave-number $k_{T C}$ corresponding to their solution in the right-hand side of (29) and expanding the terms containing the fluid velocity $U$ in series in $\theta$, we can get an approximate expression for the wave-number as

$k=k_{\mathrm{TC}} \sqrt{\frac{\exp (i \theta)+i \alpha(1-\beta \cos 4 \theta) \sin \theta \cos \theta}{1-\beta \cos 4 \theta}}$,

where $k_{T C}$ the critical wave-number for the non-isothermal binary system and $\alpha$ the parameter which defines intensity of convective flow by the external flow velocity $U$. These are

$$
\begin{aligned}
& k_{T C}=-\sqrt{\frac{V P}{2 d_{0} D_{T}}}, \quad P=\left(1+\frac{2 m C_{i}\left(1-k_{0}\right) D_{T}}{D_{C} Q / c_{p}}\right), \\
& \alpha=\frac{a U d_{0}}{4 P \rho V}\left(1+\frac{2 D_{T}}{D_{C}}-\frac{4\left(1-k_{0}\right) m C_{i} D_{T}^{2}}{P\left(Q / c_{p}\right) D_{C}^{2}}\right) .
\end{aligned}
$$

Taking into account that

$l=-\frac{\rho}{2}\left[\frac{\tan \theta}{\cos \theta}+\ln \left(\frac{1}{\cos \theta}+\tan \theta\right)\right]$

(see, among others Ref. [23]), let us rewrite the solvability condition (15) by analogy with Bouissou and Pelce [18] in the form

$$
\int_{-\infty}^{+\infty} G(\chi) \exp \left[\sqrt{C} \Psi_{\alpha}(\chi)\right] d \chi=0, \quad \chi=\tan \theta,
$$

where

$\Psi_{\alpha}(\chi)=\frac{i}{2} \int_{0}^{\chi} \frac{\left[\left(1+i \chi^{\prime}\right)\left(1+\chi^{\prime 2}\right)^{5 / 2}+i \alpha \chi^{\prime} B\left(\chi^{\prime}\right)\right]^{1 / 2} d \chi^{\prime}}{\sqrt{B\left(\chi^{\prime}\right)}}$,

$B(\chi)=\left(1+\chi^{2}\right)^{2}(1-\beta)+8 \beta \chi^{2}$,

and constant $C$ is normalized by a factor $V P \rho^{2} /\left(2 d_{0} D_{T}\right)$.

We evaluate this integral in the limit of small anisotropy by means of the method developed by Bouissou and Pelce [18]. The numerator of the integrand vanishes for $\chi$ close to $\chi=i$ (stationary phase point) and the denominator for $\chi=i(1-\sqrt{2 \beta})$ (point of singularity). As the dominant contribution to the integral is determined by the neighborhood of $\chi=i$, function $\Psi_{\alpha}(\chi)$ can be approximated by

$\Psi_{\tau}(\varphi)=2^{9 / 8} \beta^{7 / 8} \int_{1 / \sqrt{2 \beta}}^{\varphi} \frac{\left[\varphi^{\prime 7 / 2}-\tau\left(\varphi^{\prime 2}-1\right)\right]^{1 / 2}}{\sqrt{\varphi^{\prime 2}-1}} d \varphi^{\prime}$,

with

$\chi=i(1-\sqrt{2 \beta} \varphi), \quad \tau=2^{-5 / 4} \beta^{-3 / 4} \alpha$.

The integral (34) can be approximately calculated by analogy with the similar integral met in the problem of dendritic growth in a pure (one-component) system [18]. Following the result of this analysis, only two dominant contributions to the integral (34) exist: the contribution from the loop and the contribution from the stationary phase points. The first of them should be calculated between a distance $\sim \tau^{2 / 7}$ (a splitting distance of the stationary phase points) at the intersection of the steepest descent path and the real axis and $\varphi=1$. It gives an oscillating factor to the exponentially small value of the integral which behaves as $\cos \left[A_{1} \sqrt{C} \beta^{7 / 8}\left(1+B_{1} \tau^{11 / 10}\right)\right]$. Each stationary phase points contributes by a term with oscillating part of the form $\cos \left[A_{2} \sqrt{C} \beta^{7 / 8}\left(1+B_{2} \tau^{11 / 10}\right)\right]$, where $A_{1}, A_{2}, B_{1}$ and $B_{2}$ are constants. The cancelation of the sum of these contributions in Eq. (30) gives the following selected values of $C$

$C=\frac{n^{2}}{\beta^{7 / 4}}\left[1+b\left(\beta^{-3 / 4} \alpha\right)^{11 / 14}\right]$,

where $n$ is an arbitrary integer and $b$ a numerical constant. Substitution of the normalization requirement into Eq. (34) leads to the expression for the scaling factor $\sigma^{*}$ in the form

$\sigma^{*} \equiv \frac{2 d_{0} D_{T}}{\rho^{2} V}=\sigma_{0} \beta^{7 / 4}\left(1+\frac{2 D_{T}\left(1-k_{0}\right) m C_{i}}{D_{C} Q / c_{p}}\right)\left[1+b\left(\beta^{-3 / 4} \alpha\right)^{11 / 14}\right]^{-1}$,

where $\sigma_{0}$ stands for a numerical constant which can be found from the asymptotic analysis [15] or from the fitting of the model predictions to experimental data.

\section{Discussion}

Eq. (36) is the central result of our treatments. It gives a criterion for the stable mode of a dendritic tip in the presence of anisotropy of surface energy (by the parameter $\beta$ ) and for the nonisothermal binary systems under forced flow in the liquid 


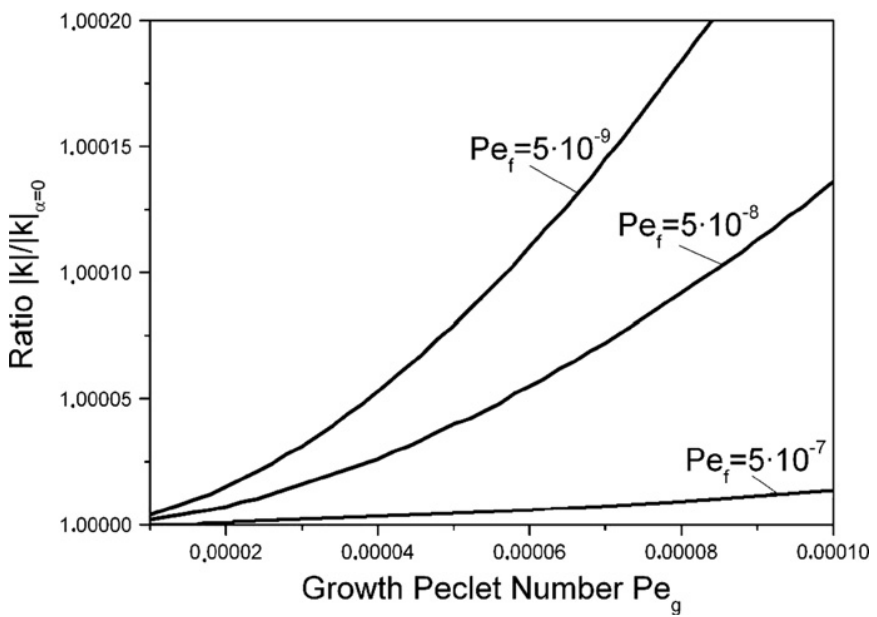

Fig. 1. Ratio for the critical wave number $|k| /|k|_{\alpha=0}$ as a function of the growth Peclet number $P_{g}=V \rho /\left(2 D_{T}\right)$ and various selected values of the flow Peclet numbers $P_{f}=U \rho /\left(2 D_{T}\right)$ as predicted by Eqs. (30) and (31).

phase. The criterion joins results obtained from the dendritic model of Bouissou and Pelce [18] for the non-isothermal pure (one-component) system with convective flow and the dendritic model of Ben Amar and Pelce [19] for non-isothermal binary stagnant system. Hence, from the criterion (36), previously obtained results on selection of stable modes in the growing tip dendrite can be summarized as follows.

\subsection{Comparison with previous models}

\subsubsection{Growth under forced convection in a pure (one-component)} system

With infinite solute diffusion, $D_{C} \rightarrow \infty$, the parameters $P$ and $\alpha$ in Eqs. (30) and (31) are equal to $P=1$ and $\alpha=a U d_{0} /(4 P \rho V)$, respectively. In this case criterion (36) transforms into the result extracted from analysis of Bouissou and Pelce (see Eq. (45) in Ref. [18]). Note that with $U=0$ and $\alpha=0$, Eq. (36) further transforms to the case of dendritic growth in a pure stagnant system [15,17].

\subsubsection{Growth in a stagnant binary system}

In Eqs. (30)-(31), the wave-number $k_{T C}$ being consistent with those one given by Ben Amar and Pelce (see Eq. (50) in Ref. [19]). With the absence of convection, i.e., at $U=0$ and $\alpha=0$, the system of Eqs. (30)-(31) completely merges with the results of Ref. [19].

\subsection{Effect of convective flow on the stable mode of dendrite tip growth}

Now, we quantitatively evaluate effect of the forced flow on the growth of the dendritic tip. It is done in examples of the critical wave-number for neutral branch of the stability curve as well as for the stability criterion for the stable mode of the dendrite growth. In calculations the following values are accepted (which are closely related to values of parameters for metallic binary systems): dimensionless ratio of the thermal diffusivity and kinematic viscosity is $D_{T} / v=10$, dimensionless ratio of the capillary constant and dendritic tip radius is $d_{0} / \rho=10^{-5}$, anisotropy parameter is $\beta=0.195$, dimensionless ratio of the thermal diffusivity and atomic diffusion coefficient is $D_{T} / D_{C}=5 \times 10^{3}$, coefficient of solute distribution is $k_{0}=0.5$, concentration in the liquid far from the interface is $C_{\infty}=1 \mathrm{at} \%$, slope of the liquidus line is $m=10 \mathrm{~K} / \mathrm{at} \%$, and ratio of the latent heat and specific heat is $Q / c_{p}=300$.

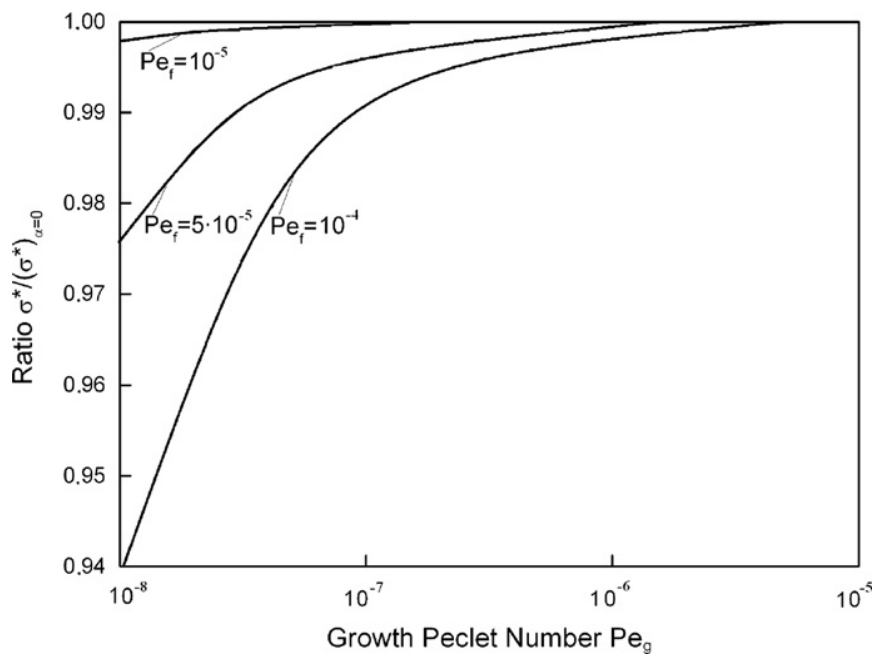

Fig. 2. Ratio $\sigma^{*} / \sigma^{*}(\alpha=0)$ as a function of the growth Peclet number $P_{\mathrm{g}}$ and various selected values of the flow Peclet numbers $P_{f}$ as predicted by Eq. (37).

First, the modulus of the critical wave number (30) can be presented as $|k| /|k|_{\alpha=0}$, where $|k|_{\alpha=0}$ is the modulus of the wave number without convection $(U=0)$ in a binary system. Fig. 1 demonstrates that with the decreasing of the intensity of flow the critical wave number increases for a fixed growth Peclet number $P_{g}$. Therefore, one can conclude that the convection shifts the critical wave number to the region of smaller $|k|$. In other words, the critical wave-length becomes larger in the presence of convection.

Second, the complete stability criterion (36) can be rewritten in the following form:

$\frac{\sigma^{*}}{\left.\sigma^{*}\right|_{\alpha=0}}=\left[1+b\left(\beta^{-3 / 4} \alpha\right)^{11 / 14}\right]^{-1}$,

with

$\left.\sigma^{*}\right|_{\alpha=0}=\sigma_{0} \beta^{7 / 4}\left(1+\frac{2 D_{T}\left(1-k_{0}\right) m C_{i}}{D_{C} Q / c_{p}}\right)$

the criterion of growth in stagnant binary system. As is shown in Fig. 2, with the decreasing the flow Peclet number $P_{f}$ and increasing of the growth Peclet number $P_{g}$ the contribution of the convection into the stability of the dendrite tip gradually decreases.

\section{Conclusions}

The two-dimensional problem of a steady-state dendritic growth with forced convective flow was taken up in a binary system. For the axi-symmetric crystal shape with anisotropy of surface energy, the analysis of a stable mode for tip of parabolic dendrite is performed. The critical wave-number is found as a marginal state at which perturbations neither grow or decay. The solvability condition gives a stability criterion (36) of an operating dendritic mode for a binary system under convective flow.

As a final note, the criterion (36) is derived for small growth Peclet numbers $P_{g}=V \rho /\left(2 D_{T}\right)$. Consequently, the present analysis of the influence of convection on dendrite growth is restricted to small growth velocity $V$ and the narrow region of the ratio $\mathrm{Pe}_{g} /$ $P e_{f}=V / U$. The extension to the region of arbitrary values of $P_{f}$ will be given in a forthcoming article. 


\section{Acknowledgments}

Authors thank Lorenz Rathke for reading manuscript and useful suggestions. D.V.A. acknowledges support by DAAD-German Academic Exchange Service under Research Program A/06/ 27363/Ref.325, Ministry of Education under the Project no. 2.1.1/ 2571, Russian Foundation for Basic Research (Grant no: 08-0100298) and Federal Target Program under the Project no. 02.740.11.0202. P.K.G. and D.M.H. acknowledge financial support from the German Research Foundation (DFG) under the Project no. HE 160/19 and DLR Agency under contract 50WM0736.

\section{References}

[1] M.C. Flemings, Solidification Processing, McGraw-Hill, New York, 1974.

[2] A.A. Chernov, In modern crystallography III, in: M. Cardona, P. Fulde, H.J. Queisser (Eds.), Springer Series in Solid-State Science, vol. 36, Springer, Berlin, 1984.

[3] W. Kurz, D.J. Fisher, Fundamentals of Solidification, third ed., Trans Tech, Aedermannsdorf, 1992.

[4] W. Kurz, R. Trivedi, Acta Metall. 38 (1990) 1.

[5] K. Eckler, D.M. Herlach, Mater. Sci. Eng. A 178 (1994) 159.

[6] W.J. Boettinger, S.R. Coriell, A.L. Greer, A. Karma, W. Kurz, M. Rappaz, R. Trivedi, Acta Mater. 48 (2000) 43.
[7] P.K. Galenko, O. Funke, J. Wang, D.M. Herlach, Mater. Sci. Eng. 375-377 (2004) 488.

[8] O. Funke, G. Phanikumar, P.K. Galenko, L. Chernova, S. Reutzel, M. Kolbe, D.M. Herlach, J. Cryst. Growth 297 (2006) 211.

[9] D.M. Herlach, P. Galenko, D. Holland-Moritz, Metastable Solids from Undercooled Melts, Elsevier, Amsterdam, 2007.

[10] M. Asta, C. Beckermann, A. Karma, W. Kurz, R. Napolitano, M. Plapp, G. Purdy, M. Rappaz, R. Trivedi, Acta Mater. 57 (2009) 941.

[11] G.P. Ivantsov, Dokl. Akad. Nauk SSSR 58 (1947) 567.

[12] W.W. Mullins, R.F. Sekerka, J. Appl. Phys. 35 (1964) 444

[13] J.S. Langer, H. Müller-Krumbhaar, Acta Metall. 26 (1978) 1681.

[14] D.A. Kessler, J. Koplik, H. Levine, Adv. Phys. 37 (1988) 255.

[15] E. Brener, V.I. Melnikov, Adv. Phys. 40 (1991) 53.

[16] M. Ben-Amar, Ph. Bouisou, P. Pelce, J. Cryst. Growth 92 (1988) 97.

[17] P. Pelce, Dynamics of Curved Fronts, Academic Press, Boston, 1988.

[18] Ph. Bouissou, P. Pelce, Phys. Rev. A 40 (1989) 6673.

[19] M. Ben Amar, P. Pelce, Phys. Rev. A 39 (1989) 4263

[20] P.K. Galenko, D.M. Herlach, in: D.M. Herlach (Ed.), Phase Transformations in Multicomponent Systems, Wiley-VCH, Weinheim, 2008, pp. 353-373.

[21] J. Wilkinson, Quart. J. Mech. Appl. Math. VIII (1955) 415.

[22] S.K. Dash, W.N. Gill, Int. J. Heat Mass Transfer 27 (1984) 1345

[23] P. Pelce, D. Bensimon, Nucl. Phys. B 2 (1987) 259.

[24] Ya. Zel'dovich, A.G. Istratov, N.I. Kidin, V.B. Librovich, Combust. Sci. Technol. 24 (1980) 1.

[25] B. Caroli, C. Caroli, B. Roulet, J.S. Langer, Phys. Rev. A 33 (1986) 442.

[26] B.I. Shraiman, Phys. Rev. Lett. 56 (1986) 2028.

[27] T. Dombre, V. Hakim, Y. Pomeau, C. R. Acad. Sci. 302 (1986) 803. 